\title{
LIMIAR ANAERÓBIO EM ATLETAS DE NATAÇÃO: UMA REVI- SÃO SISTEMÁTICA
}

\author{
Jhonny Kleber Ferreira da Silva \\ Universidade Federal do Paraná, Curitiba, Paraná, Brasil. \\ Alysson Afonso Nadalin Enes \\ Universidade Federal do Paraná, Curitiba, Paraná, Brasil. \\ Poliana de Lima Costa \\ Universidade Federal do Paraná, Curitiba, Paraná, Brasil. \\ Danilo Leonel Alves \\ Universidade Federal do Paraná, Curitiba, Paraná, Brasil. \\ Rafael Octaviano de Souza \\ Universidade Federal do Paraná, Curitiba, Paraná, Brasil. \\ Raul Osiecki \\ Universidade Federal do Paraná, Curitiba, Paraná, Brasil.
}

\begin{abstract}
Resumo
Este artigo objetivou realizar uma revisão sistemática, relatando os métodos utilizados na literatura para a identificação do limiar anaeróbio em nadadores e, além disso, verificar a existência de possíveis lacunas que justifiquem a realização de novos estudos. Para isso, buscou-se artigos nas bases de dados eletrônicas Pubmed, Web Of Science, SciELO e Science Direct.O Protocolo de $200 \mathrm{~m}$ mostrou-se o mais utilizado, indicando ser o mais adequado para grupos de provas distintas. Porém, constatou-se que a literatura carece de delineamentos adequados para cada estilo de nado, assim como para a especialidade de cada atleta. Conclui-se que é possível utilizar diferentes métodos para identificação do limiar anaeróbio e empregá-los na prescrição e controle do treinamento de nadadores.
\end{abstract}

Palavras-chave: Natação. Limiar Anaeróbio. Aptidão Física.

\section{ANAEROBIC THRESHOLD IN SWIMMING ATHLETES: A SYSTEMATIC REVIEW}

\begin{abstract}
The objective of this systematic review was to report the methods used in the literature for the identification of the anaerobic threshold in swimmers and to verify the existence of possible gaps that justify the realization of new studies. Articles were searched in the electronic databases of Pubmed, Web Of Science, SciELO e Science Direct. The $200 \mathrm{~m}$ Protocol is the most used, indicating that it is the most appropriate for different test groups. However, we found that the literature lacks adequate designs for each style of swimming, as well as for the specialty of each athlete. It is concluded that it is possible to use different methods to identify the anaerobic threshold, providing its use for the prescription and control of training of swimmers.
\end{abstract}


Keywords: Swimming. Anaerobic threshold. Physical fitness.

\title{
UMBRAL ANAEROBIO EN ATLETAS DE NATACIÓN: UNA REVISIÓN SIS- TEMÁTICA
}

\begin{abstract}
Resumen
El objetivo de esta revisión sistemática fue describirlos métodos utilizados en la literatura para la identificación del umbral anaeróbico en nadadores y, verificar la existencia de posibles lagunas que justifiquen la realización de nuevos estudios. Se buscaron artículos en las bases de datos electrónicas Pubmed, Web of Science, SciELO y Science Direct. El protocolo de $200 \mathrm{~m}$ fue el más utilizado, lo que indica que es el más adecuado para grupos de pruebas distintas. Se constató que la literatura carece de delineamientos para cada estilo de nado, así como para la especialidad de los atletas. Se concluye que es possible utilizar diferentes métodos para identificar el umbral anaeróbico, proporcionando el uso de es te para la prescripción y control del entrenamiento de nadadores.
\end{abstract}

Palabras clave: Natación. Umbral anaeróbico. Aptitud física.

\section{Introdução}

As demandas fisiológicas da natação possuem especificidade em relação a outras modalidades por ser praticada em meio aquático, ocasionando diferentes respostas fisiológicas frente ao exercício (GRAEF \& KRUEL, 2006). Consequentemente, algumas limitações podem surgir, como a dificuldade em determinar a contribuição relativa de produção de energia pelo metabolismo aeróbio e anaeróbio durante o exercício (MAGLISCHO, 1999). Diversos métodos têm sido usados para identificar a resposta do lactato sanguíneo e do limiar anaeróbio (LAn), frequentemente utilizado para avaliações da capacidade de trabalho aeróbio e anaeróbio, assim como para prescrição e acompanhamento do treinamento físico (GRECO et al., 2010; GOBATTO et al., 2001). Porém, a utilização de métodos diretos de avaliação do LAn em atletas de natação pode ser limitada por questões financeiras ou pela falta de praticidade na aquisição de dados (HOEFELMANN et al., 2011). Sendo assim, existem métodos indiretos para predição do LAn, como protocolos validados que utilizam variáveis não invasivas, como a velocidade crítica (VC) (KOKUBUN et al., 1996), força crítica (PAPOTI et al., 2001), variabilidade da frequência cardíaca (DI MICHELE et al., 2012), tolerância à acidose (DEMINICE et al., 2007) e teste incremental no ergômetro de braço (DENADAI et al., 2002).

Portanto, diferentes protocolos e critérios são utilizados para a determinação dessa variável em atletas nadadores, o que tem aumentado o interesse pela validação de protocolos de baixo custo e com boa validade ecológica. A diferença cronológica e a diversidade de métodos para encontrar o LAn em nadadores, em determinados casos, não levam em consideração a especialidade do atleta em diferentes estilos de nado. Devido à existência de diferentes protocolos de teste na natação, torna-se difícil identificar qual se adéqua melhor para determinar o LAn em nadadores em diferentes distâncias e estilos. Desta forma, a presente revisão tem como objetivo relatar os métodos para a identificação do LAn em nadadores e verificar a existência de possíveis lacunas que justifiquem a realização de novos estudos. 


\section{Metodologia}

A revisão sistemática é um tipo de investigação científica que tem por objetivo reunir, avaliar criticamente e conduzir um apanhado dos resultados de diversos estudos primários sobre um determinado tema, visando a descobrir lacunas na literatura para realização de futuros estudos (SAMPAIO \& MANCINI, 2007; COOK, MULROW, HAYNES, 1997).

Para a realização do presente estudo, foi identificado o problema e o objetivo da revisão, bem como a base de dados e os descritores. Em seguida, procedeu-se à busca, em inglês e português, dos descritores lactate threshold (limiar de lactato), anaerobic threshold (limiar anaeróbio), swimmers (nadadores) e competitive swimming (natação competitiva) nas bases de dados eletrônicas Pubmed, Web Of Science, SciELO, Science Direct. Posteriormente, optou-se pela utilização do termo anaerobic threshold and competitive swimming. Essa primeira busca foi efetuada pelos autores em momentos distintos e não se delimitou datas para inclusão dos artigos.

\section{Critérios de avaliação da qualidade metodológica dos artigos}

Alguns estudos utilizaram modelos de pontuação para determinar a qualidade metodológica dos artigos (PAZA et al., 2017; ZOLTOWSKI et al., 2014). Para essa revisão, foi adotado um protocolo específico adaptado, elaborado pela literatura (SANTOS et al., 2014). Em uma reunião de consenso, os autores definiram os critérios de inclusão e exclusão dos estudos. A qualidade dos artigos foi verificada da seguinte forma: (1) apresenta características gerais da amostra com desvio padrão das idades; (2) apresenta metodologia compatível com os objetivos da presente revisão; (3) apresenta descrição do método utilizado para a determinação do LAn; (4) apresenta valores do limiar de lactato nos resultados do estudo; (5) apresenta conclusão que responda ao objetivo do estudo (Tabela 1). Os autores estabeleceram que os artigos analisados deveriam ter, no mínimo, três pontos para estar dentro da qualidade metodológica e serem incluídos.

\section{Resultados}

Os artigos encontrados estavam distribuídos, especificamente, nas seguintes bases de dados: PubMed: 189 artigos em inglês e zero em português; Science Direct: 1903 em inglês e dois em português; BVS: 218 em inglês e 21 em português; Scopus: 3.707 em inglês e 31 em português; Web of Science: nenhum artigo foi encontrado. Foram totalizados 6.017 artigos em inglês e 54 artigos em português, conforme mostra a Figura 1. Posteriormente, foram eliminados os artigos repetidos, restando 2.751. Em seguida, foram excluídos os artigos não condizentes com o tema, restando 47 estudos para a leitura dos resumos, em reunião de consenso. Após esta etapa do processo, 29 trabalhos foram dispensados, respeitando-se os seguintes critérios: 19 artigos que não objetivaram identificar o LAn (ANDERSON et al., 2003; COSTA et al., 2012; AVLONITOU, 1996; GREENWOOD et al., 2008; KONSTANTAKI, SWAINE, 1999; SANTHIAGO et al., 2009; CLEMENTE-SUÁREZ et al., 2017; COSTA, et al., 2010; CHATARD, MUJIKA, 1999; ESPADA et al., 2015; SMITH, MCMURRAY, SYMANSKI, 1984; GONDIM et al., 2007; SANTHIAGO et al., 2011; NEIVA, FERNANDES, VILASBOAS, 2011; SOARES et al., 2014; BARON, PELAYO, 2001; REILLY, WOODBRIDGE, 1999; CAMPOS et al., 2012; LI et al., 2012). Além desses, um estudo com indivíduos não atletas (SOULTANAKIS, MANDALOUFAS, PLATANOU, 2012), 
cinco estudos com métodos que não foram realizados em piscina (MERCIER et al., 1993; LONGO, ALMEIDA, ÃVILA, 1995; RIBEIRO, GALDINO, BALIKIAN, 2017; ALEXIOU et al., 1998; GACESA, BARAK, GRUJIC, 2009), três artigos de revisão (OLBRECHT, 2011; TREFFENE et al., 1980; TOUBEKIS, TOKMAKIDIS, 2013), um artigo em que a faixa etária está fora do critério de inclusão (MEJIAS et al., 2014).

Em última etapa, de acordo com os critérios de qualidade, foi excluído um artigo (CELLINI et al., 1986) por ele não atender a pontuação requerida, pois, fora estabelecido que os artigos deveriam ter, no mínimo, três pontos para estar dentro da qualidade metodológica. Desta forma, utilizou-se 17 artigos para o estudo, organizados por ordem cronológica, contemplando os seguintes elementos: autor e ano de publicação, descrição da amostra, métodos e valores médios das concentrações de lactato, conforme a Tabela 2 .

Figura 1. Etapas da revisão sistemática
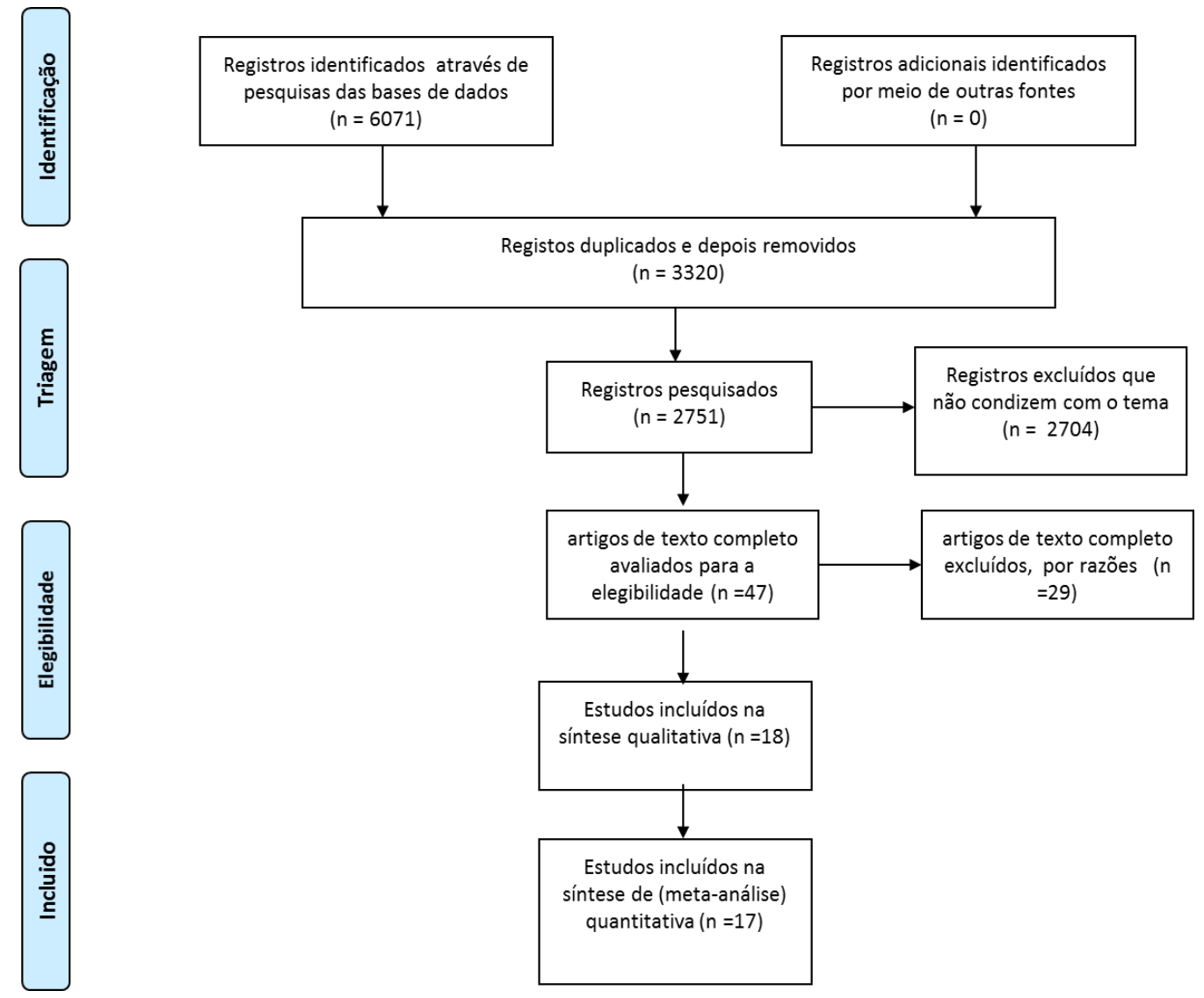

A tabela abaixo demonstra a pontuação individual de qualidade metodológica de cada artigo analisado. 
Tabela 1. Qualidade metodológica dos artigos selecionados

\begin{tabular}{|c|c|c|c|c|c|c|c|}
\hline Autor & Artigos & 1 & 2 & 3 & 4 & 5 & Total \\
\hline $\begin{array}{lll}\text { Cellini et } a l ., \\
1986\end{array}$ & $\begin{array}{l}\text { Noninvasive Determination of the Anaerobic Threshold in } \\
\text { Swimming }\end{array}$ & $\mathrm{x}$ & $\mathrm{x}$ & & & & 2 \\
\hline $\begin{array}{l}\text { Kokubun et al., } \\
1996\end{array}$ & $\begin{array}{l}\text { Velocidade crítica como estimador do limiar anaeróbico na } \\
\text { natação }\end{array}$ & & $\mathrm{X}$ & $\mathrm{x}$ & $\mathrm{X}$ & $\mathrm{X}$ & 4 \\
\hline $\begin{array}{l}\text { Papoti et al., } \\
2001\end{array}$ & Aerobic and anaerobic performances in tethered swimming & $\mathrm{x}$ & $\mathrm{X}$ & $\mathrm{x}$ & $\mathrm{X}$ & & 4 \\
\hline $\begin{array}{l}\text { Pyne, Lee, } \\
\text { Swanwick, 2001 }\end{array}$ & Monitoring the lactate threshold in world ranked swimmers & $\mathrm{x}$ & $\mathrm{X}$ & $\mathrm{x}$ & $\mathrm{X}$ & & 4 \\
\hline $\begin{array}{l}\text { Denadai et al., } \\
2002\end{array}$ & $\begin{array}{l}\text { Efeitos de oito semanas de treinamento de natação no limiar } \\
\text { anaeróbio determinado na piscina e no ergômetro de braço }\end{array}$ & $\mathrm{X}$ & $\mathrm{X}$ & $\mathrm{X}$ & & $\mathrm{X}$ & 4 \\
\hline $\begin{array}{l}\text { Dekerle et al., } \\
2002\end{array}$ & $\begin{array}{l}\text { Validity and Reliability of Critical Speed, Critical Stroke Rate, } \\
\text { and Anaerobic Capacity in Relation to Front Crawl Swimming } \\
\text { Performances }\end{array}$ & $\mathrm{x}$ & $\mathrm{x}$ & $\mathrm{X}$ & & & 3 \\
\hline Gatti et al., 2004 & $\begin{array}{l}\text { Respostas fisiológicas e biomecânicas de nadadores em diferen- } \\
\text { tes intensidades de nado }\end{array}$ & & & $\mathrm{x}$ & $\mathrm{x}$ & $\mathrm{x}$ & 3 \\
\hline $\begin{array}{l}\text { Ribeiro et al., } \\
2004\end{array}$ & $\begin{array}{l}\text { Limiar anaeróbio em natação: comparação entre diferentes pro- } \\
\text { tocolos }\end{array}$ & $\mathrm{X}$ & $\mathrm{x}$ & $\mathrm{x}$ & $\mathrm{x}$ & $\mathrm{x}$ & 5 \\
\hline $\begin{array}{l}\text { Papoti et al., } \\
2005\end{array}$ & $\begin{array}{l}\text { Utilização do intercepto-y na avaliação da aptidão anaeróbia e } \\
\text { predição da performance de nadadores treinados }\end{array}$ & $\mathrm{X}$ & & $\mathrm{x}$ & $\mathrm{x}$ & $\mathrm{X}$ & 4 \\
\hline Lima et al., 2006 & $\begin{array}{l}\text { Proposta de teste incremental baseado na percepção subjetiva de } \\
\text { esforço para determinação de limiares metabólicos e parâmetros } \\
\text { mecânicos do nado livre }\end{array}$ & $\mathrm{x}$ & & $\mathrm{x}$ & $\mathrm{x}$ & & 3 \\
\hline $\begin{array}{l}\text { Anderson et al., } \\
2006\end{array}$ & $\begin{array}{l}\text { Monitoring seasonal and long-term changes in test performance } \\
\text { in elite swimmers }\end{array}$ & & & $\mathrm{X}$ & $\mathrm{x}$ & $\mathrm{X}$ & 3 \\
\hline $\begin{array}{l}\text { Deminice et al., } \\
2007\end{array}$ & $\begin{array}{l}\text { Série de treinamento intervalado de alta intensidade como índice } \\
\text { de determinação da tolerância à acidose na predição da perfor- } \\
\text { mance anaeróbia de natação }\end{array}$ & $\mathrm{x}$ & $\mathrm{X}$ & $\mathrm{X}$ & $\mathrm{X}$ & $\mathrm{x}$ & 5 \\
\hline $\begin{array}{l}\text { Hoefelmann et } \\
\text { al., } 2011\end{array}$ & $\begin{array}{l}\text { Comparação de diferentes métodos para identificação do limiar } \\
\text { anaeróbio em nadadores }\end{array}$ & $\mathrm{x}$ & $\mathrm{X}$ & $\mathrm{X}$ & $\mathrm{x}$ & $\mathrm{x}$ & 5 \\
\hline $\begin{array}{l}\text { Machado et al., } \\
2011\end{array}$ & $\begin{array}{l}\text { Effect of } 12 \text { weeks of training on critical velocity and maximal } \\
\text { lactate steady state in swimmers }\end{array}$ & $\mathrm{x}$ & $\mathrm{X}$ & $\mathrm{x}$ & & $\mathrm{x}$ & 4 \\
\hline $\begin{array}{l}\text { Di Michele et } \\
\text { al., } 2012\end{array}$ & $\begin{array}{l}\text { Estimation of the anaerobic threshold from heart rate variability } \\
\text { in an incremental swimming test }\end{array}$ & $\mathrm{x}$ & $\mathrm{x}$ & $\mathrm{x}$ & $\mathrm{x}$ & $\mathrm{x}$ & 5 \\
\hline $\begin{array}{l}\text { Skorski et al., } \\
2012\end{array}$ & Intensity control in swim training by means of the individual & $\mathrm{X}$ & $\mathrm{X}$ & $\mathrm{X}$ & $\mathrm{X}$ & $\mathrm{x}$ & 5 \\
\hline $\begin{array}{l}\text { Campos et al., } \\
2014\end{array}$ & $\begin{array}{l}\text { The response of the lactate minimum test to a } 12 \text {-week swim- } \\
\text { ming training }\end{array}$ & $\mathrm{X}$ & $\mathrm{X}$ & $\mathrm{X}$ & $\mathrm{X}$ & $\mathrm{x}$ & 5 \\
\hline $\begin{array}{l}\text { Kalva-Filho } \\
\text { al., } 2015\end{array}$ & $\begin{array}{l}\text { Relationship of aerobic and anaerobic parameters with } 400 \mathrm{~m} \\
\text { front crawl swimming performance }\end{array}$ & $\mathrm{x}$ & $\mathrm{X}$ & $\mathrm{X}$ & $\mathrm{x}$ & & 4 \\
\hline
\end{tabular}

Nota: Critérios de qualidade: (1) apresenta as características gerais da amostra com desvio padrão das idades; (2) apresenta metodologia compatível com os objetivos da presente revisão; (3) apresenta descrição do método utilizado para a determinação do LAn; (4) apresenta valores do limiar de lactato nos resultados do estudo; (5) apresenta conclusão que responda ao objetivo do estudo.

\section{Objetivos propostos}

Quanto aos objetivos dos estudos, buscou-se investigar, em propostas variadas, o desempenho físico (HOEFELMANN et al., 2011), a análise biomecânica (GATTI et al., 2004) e os efeitos do treinamento em atletas nadadores durante semanas (MACHADO et al., 2011). Alguns artigos encontram o LAn a partir da análise de outras variáveis, enquanto outros objetivaram identificar o LAn de forma direta. Embora os artigos selecionados para esta revisão sistemática tenham tido objetivos diferentes, todos eles identificaram o LAn em determinada fase do estudo. 


\section{Metodologia e protocolos de identificação do limiar anaeróbio}

Verificou-se diversas formas de identificação do LAn em diferentes protocolos de testes. Os protocolos de testes incrementais foram os mais utilizados (KOKUBUN et al., 1996; PAPOTI et al., 2001; PYNE, LEE, SWANWICK, 2001; GATTI et al., 2004; LIMA et al., 2006; ANDERSON et al., 2006; DEMINICE et al., 2007; HOEFELMANN et al., 2011; MACHADO et al., 2011; DI MICHELE et al., 2012; CAMPOS et al., 2014). Observou-se também diferentes períodos de coleta de lactato, sendo divididos em sessão de horas (RIBEIRO et al., 2004; LIMA et al., 2006) ou semanas (CAMPOS et al., 2014). De forma geral, podemos citar métodos de 30 minutos (DENADAI et al., 2002), VC (KOKUBUN et al., 1996; DEKERLE et al., 2002; HOEFELMANN et al., 2011; MACHADO et al., 2011) e teste contínuos (RIBEIRO et al.,2004; SKORSKI et al., 2012) que também foram utilizados para identificação do LAn (PAPOTI et al., 2001; PYNE, LEE, SWANWICK, 2001; GATTI et al., 2004; RIBEIRO et al., 2004; LIMA et al., 2006; ANDERSON et al., 2006; DEMINICE et al., 2007; HOEFELMANN et al., 2011; DI MICHELE et al., 2012; SKORSKI et al., 2012, CAMPOS et al., 2014).

Constatou-se que o protocolo de distância de repetições de $200 \mathrm{~m}$ foi o mais usado nos artigos analisados, sendo esta distância a mais utilizada na identificação do LAn para o grupo de nadadores descrito nos artigos (DEKERLE et al., 2002; RIBEIRO et al., 2004; PAPOTI et al., 2005; LIMA et al., 2006; MACHADO et al., 2011; SKORSKI et al., 2012). Outras distâncias foram aplicadas nos testes, dentre estas, $50 \mathrm{~m}$, $100 \mathrm{~m}, 400 \mathrm{~m}$ e teste de 30 minutos, considerando diferentes intensidades para cada protocolo (PAPOTI et al., 2001; PYNE, LEE, SWANWICK, 2001; GATTI et al., 2004; RIBEIRO et al., 2004; LIMA et al., 2006; ANDERSON et al., 2006; DEMINICE et al., 2007; HOEFELMANN et al., 2011; DI MICHELE et al., 2012; SKORSKI et al., 2012, CAMPOS et al., 2014; DEKERLE et al., 2002; PAPOTI et al., 2005; LIMA et al., 2006; MACHADO et al., 2011).

\section{Resultados da qualidade metodológica}

Ao relatar a descrição das amostras (Critério 1), três artigos não atenderam o critério de qualidade da amostra, por não apresentarem os dados de média de idade e desvio padrão do grupo de nadadores (KOKUBUN et al., 1996; GATTI et al., 2004; ANDERSON et al., 2006). Na metodologia (Critério 2), quatro artigos não atenderam aos critérios estabelecidos, pois não apresentaram descrito em seus métodos a identificação do LAn. Porém, durante o estudo, tais artigos possibilitavam essa identificação de forma indireta (GATTI et al., 2004; PAPOTI et al., 2005; LIMA et al., 2006; ANDERSON et al., 2006).

A descrição do método (Critério 3) utilizado para determinar o LAn, esteve presente nos 17 artigos. Ao relatar valores médios das concentrações de lactato (Critério 4) presentes nos estudos, apenas três artigos não demonstraram ou expuseram os valores médios das concentrações de lactato em sua descrição (DENADAI et al., 2002; DEKERLE et al., 2002; MACHADO et al., 2011). Ao se reportar às conclusões (Critério 5), apenas seis artigos não concluíram de maneira efetiva os objetivos propostos (PAPOTI et al., 2001; PYNE, LEE, SWANWICK, 2001; DEKERLE et al., 2002; LIMA et al., 2006; KALVA-FILHO et al., 2015). 


\section{Principais resultados encontrados} dos.

A Tabela 2 apresenta as metodologias e os valores médios de lactato encontra-

Tabela 2. Metodologia dos artigos selecionados

\begin{tabular}{|c|c|c|c|}
\hline Autor & Amostra & Método & $\begin{array}{l}\text { Valores médios } \\
\text { de Lactato }\end{array}$ \\
\hline $\begin{array}{l}\text { Kokubun et } \\
\text { al., (1996) }\end{array}$ & $\begin{array}{l}48 \text { nada- } \\
\text { dores }\end{array}$ & $\begin{array}{l}\text { LAn: } 3 \times 200 m \text { a } 85 \%, 90 \% \text { e } 95 \% \text { da Vmáx } \\
\text { VC: } 100,200 \text { e } 400 \text { m máximos } \\
\text { LAn x VC: 3rep } 5 \times 400 m \text { a } 100 \%, 102 \% \text { e } \\
104 \% \text { da VC }\end{array}$ & $6.69 \pm 1.68 \mathrm{mmol}$ \\
\hline $\begin{array}{l}\text { Papoti et } \\
\text { al., (2001) }\end{array}$ & $\begin{array}{l}12 \text { nada- } \\
\text { dores }(3 \mathrm{~F} \text {, } \\
9 \mathrm{M})\end{array}$ & $\begin{array}{l}\text { Teste incremental de nado atado. Carga Inici- } \\
\text { al: } 20 \mathrm{~N} \text {, com incrementos de } 10 \mathrm{~N} \text { a cada } 3 \\
\text { minutos, e descanso de } 30 \text { segundos }\end{array}$ & $11.03 \pm 2.0 \mathrm{mmol}$ \\
\hline $\begin{array}{l}\text { Pyne, Lee, } \\
\text { Swanwick, } \\
\text { (2001) }\end{array}$ & $\begin{array}{l}12 \text { nada- } \\
\text { dores }(8 \mathrm{M} \\
\text { e } 4 \mathrm{~F})\end{array}$ & Teste incremental, $7 \times 200 \mathrm{~m}$ & $\begin{array}{l}3.6 \pm 0.2 \mathrm{mmol} \mathrm{em} \\
\text { Janeiro; } \\
3.2 \pm 0.1 \mathrm{mmol} \mathrm{em} \\
\text { Maio; } \\
2.9 \pm 0.2 \mathrm{mmol} \mathrm{em} \\
\text { Julho; } \\
3.4 \pm 0.2 \mathrm{mmol} \mathrm{em} \\
\text { Agosto }\end{array}$ \\
\hline $\begin{array}{l}\text { Denadai et } \\
\text { al., (2002) }\end{array}$ & $\begin{array}{l}14 \text { nada- } \\
\text { dores GE } \\
=(5 \mathrm{M} \mathrm{e} \\
2 \mathrm{~F}) \mathrm{GC}= \\
(6 \mathrm{M} \text { e } 3 \mathrm{~F})\end{array}$ & $\begin{array}{l}\text { LAn (natação): Teste intervalado a } 85 \% \text { e } \\
\text { outro a } 95 \% \text { da Vmáx dos } 400 \mathrm{~m} \text {, com inter- } \\
\text { valo de } 30 \text { minutos } \\
\text { LAn (ergômetro): Teste progressivo contínuo } \\
\text { no ergômetro de braço, com rotação fixa de } 60 \\
\text { rpm, com carga inicial de } 33,3 \text { Watts, e incre- } \\
\text { mentos de } 16,6 \text { Watts a cada } 3 \text { minutos }\end{array}$ & - \\
\hline $\begin{array}{l}\text { Dekerle et } \\
\text { al., (2002) }\end{array}$ & $\begin{array}{l}10 \text { nada- } \\
\text { dores }(8 \mathrm{M} \\
\text { e } 2 \mathrm{~F})\end{array}$ & $\begin{array}{l}\text { Teste de } 30 \text { minutos. Em seguida tiros de } 50 \\
\mathrm{~m}, 100 \mathrm{~m}, 200 \mathrm{~m} \text { e } 400 \mathrm{~m} .\end{array}$ & - \\
\hline $\begin{array}{l}\text { Gatti et al., } \\
\text { (2004) }\end{array}$ & $\begin{array}{l}7 \text { nadado- } \\
\text { res }(6 \mathrm{M} \mathrm{e} \\
1 \mathrm{~F})\end{array}$ & $\begin{array}{l}8 \times 200 \text { m, em nado livre, com velocidades de } \\
80 \%, 85 \%, 90 \%, 95 \% \text { e } 100 \% \text { do percentual } \\
\text { de esforço de cada nadador }\end{array}$ & 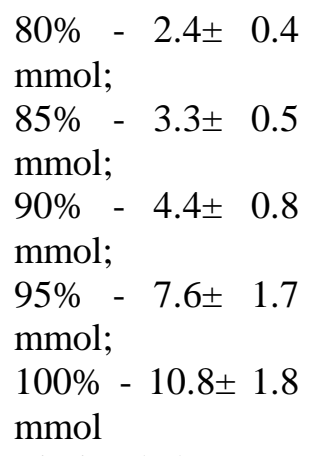 \\
\hline $\begin{array}{l}\text { Ribeiro et } \\
\text { al., (2004) }\end{array}$ & $\begin{array}{l}10 \text { nada- } \\
\text { dores }\end{array}$ & $\begin{array}{l}2000 \mathrm{~m} \text { em estilo crawl; teste incremental de } \\
200 \mathrm{~m} \text {, com incrementos de velocidade; } \\
2 \times 50 \mathrm{~m} \text { em estilo crawl com intervalos de } 1 \\
\text { minuto }\end{array}$ & $\begin{array}{l}\text { Limiar de lactato - } \\
3.41 \pm 1.38 \mathrm{mmol} \text {; } \\
\text { lactato mínimo - } \\
7.05 \pm 2.52 \mathrm{mmol} \\
\text { velocidade da fase } \\
\text { máxima estável } \\
\text { de lactato (MLSS) } \\
-4.41 \pm 1.27 \mathrm{mmol} \text {. }\end{array}$ \\
\hline
\end{tabular}


$\begin{array}{lll}\begin{array}{l}\text { Papoti et } \\ \text { al., (2005) }\end{array} & \begin{array}{l}14 \text { nada- } \\ \text { dores, } \\ (11 \mathrm{M}\end{array} & \begin{array}{l}\text { Teste de nado atado em piscina de } 25 \mathrm{~m}: 100 \\ \mathrm{~m}, 200 \mathrm{~m}, 300 \mathrm{~m}, 400 \mathrm{~m} \text { e } 600 \mathrm{~m} \text { em estilo }\end{array} \\ & 3 \mathrm{~F})\end{array}$

Lima et al., 12 nada- Tiros máximos nas distâncias de 200 m e 400 (2006) dores $\quad \mathrm{m}$ $(12 \mathrm{M})$ m Teste incremental com 5 tiros de $200 \mathrm{~m}$, com intervalo de 90 segundos entre os tiros

Anderson et 40 nada- Teste incremental. 7x200 m $\begin{array}{ll}\text { al., (2006) } & \begin{array}{l}\text { dores } \\ (24 \mathrm{M} \\ 16 \mathrm{~F})\end{array}\end{array}$

Deminice et 10 nadaal., (2007) dores (8M e $2 \mathrm{~F}$ )

Hoefelmann 9 nadadoet al., (2011) res (9M)

Machado et 10 nadaal., (2011) dores $(10 \mathrm{M})$

Três dias de testes em nado crawl

VLAn: 3×100 m a 85\%, 90\% e 95\% da Vmáx, com intervalo de 3 minutos

LAn (4 mmol): $3 \times 400 \mathrm{~m}$ no nado crawl, a $80 \%, 85 \%$ e $90 \%$ da Vmáx

VC: tempo nos $200 \mathrm{~m}, 400 \mathrm{~m}$ e $700 \mathrm{~m}$

VC: $50 \mathrm{~m}, 100 \mathrm{~m}, 200 \mathrm{~m}$ e $400 \mathrm{~m}$, com intervalo de $24 \mathrm{~h}$

MLSS: 3 séries de $4 \times 400 \mathrm{~m}$, correspondentes a $98 \%, 100 \%$ e $102 \%$ da VC, com intervalos de 45-60 segundos

$\begin{array}{ll}\text { Di Michele } & 14 \text { nada- } \\ \text { et al., (2012) } & \text { dores }(6 \mathrm{M} \\ & \text { e 8F) }\end{array}$

Teste incremental, 7x200 m em nado crawl

Skorski et 18 nadaal., (2012) dores $(13 \mathrm{M}$ $5 \mathrm{~F})$

$$
200
$$

$200 \mathrm{~m}$, sendo, o tempo, reduzido em 10 segundos por repetição. Para cada repetição, e foram adotados intervalos de 45 segundos

$6.80 \pm 1.03 \mathrm{mmol}$.

$\cong 8,50 \pm 2.0 \mathrm{mmol}$ (no último estágio do teste)

$10.5 \pm 2.1 \mathrm{mmol}$ (Masculino) e $9.2 \pm 1.9 \mathrm{mmol}$ (Feminino)

$13.5 \pm 1.6 \mathrm{mmol}$

$4.3 \pm 2.2 \mathrm{mmol}$

$-$

$8.2 \pm 2.0 \mathrm{mmol}$ (no sétimo estágio do teste)

20x100 m - 1.8

$\mathrm{mmol}$;

$5 \times 400 \quad m-4.4$

mmol;

$5 \times 200 \mathrm{~m}-6.3$

mmol;

10x100 m - 5.8

$\mathrm{mmol}$

T0 - 9.79 \pm 3.01

mmol; T4 -

$9.90 \pm 3.46 \mathrm{mmol}$;

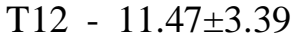
mmol

$8.6 \pm 3.2 \mathrm{mmol}$

Kalva-Filho 22 nada- Teste de lactato mínimo para determinar a et al., (2015) dores velocidade mínima de lactato no protocolo de $(15 \mathrm{M} \quad$ e $400 \mathrm{~m}$ em nado crawl $7 \mathrm{~F})$ 


\section{Discussão}

Os resultados do estudo mostraram diferentes métodos utilizados na identificação do LAn. De acordo com os artigos analisados, não há um método habitual para os diferentes objetivos. No artigo de Kokubun et al. (1996) que teve por objetivo verificar se a VC corresponde a concentração de lactato sanguíneo, os autores identificaram uma correlação positiva forte $(r=0,89)$ entre LAn e VC. Em outro artigo, Hoefelmann et al. (2011) comparou diferentes métodos utilizados para predizer a fase máxima estável de lactato (MLSS) e verificou a correlação entre VC e LAn. Os resultados encontrados pelos autores demonstraram que métodos diretos e indiretos para determinação do LAn $(4 \mathrm{mmol})$ possuem correlações positivas e significativas (HOEFELMANN et al., 2011). Esses estudos indicam que a VC é um método confiável para predizer o LAn em nadadores.

Outras formas de identificação do LAn também foram abordadas, utilizando métodos alternativos, como no estudo de Lima et al. (2006), que buscou estimar o limiar metabólico a partir de métodos lactacidêmicos em um teste incremental baseado na percepção subjetiva de esforço, além de verificar se a capacidade de impulsão influencia na performance aeróbia e anaeróbia (LIMA et al., 2006; PAPOTI et al., 2005).

Adicionalmente, artigos que identificaram o LAn em diferentes momentos da periodização também foram encontrados. Eles são relevantes para prescrição do treinamento do nadador durante a temporada, pois verificam o comportamento da concentração de lactato ao longo de vários períodos de tempo e reporta-se como uma ferramenta importante na prescrição de treino dos atletas (PYNE, LEE, SWANWICK, 2001; CAMPOS et al., 2014). O maior valor de concentração de lactato encontrado foi de $13.5 \pm 1.6 \mathrm{mmol}$, no estudo do autor Deminice et al. (2007), que teve como objetivo determinar a tolerância à acidose através de uma série de nados intervalados de alta intensidade e relacionar com variáveis fisiológicas e mecânicas para predição da performance de $100 \mathrm{~m}$ na natação. Contudo, os demais estudos apresentaram diferentes valores da concentração de lactato em diferentes métodos, sendo 1.8 mmol o valor mais baixo (em 20x100 m) (SKORSKI et al., 2012). Desta maneira, é necessário identificar em quais valores se enquadram melhor cada atleta e prova em disputa (velocidade, meio fundo ou fundo).

É importante destacar que a metragem mais utilizada nos protocolos de teste foi de $200 \mathrm{~m}$. Essa metragem parece estar melhor caracterizada tanto para grupos de nadadores velocistas quanto para meio fundistas (KOKUBUN et al., 1996; PYNE, LEE, SWANWICK, 2001; DEKERLE et al., 2002; GATTI et al., 2004; LIMA et al., 2006; ANDERSON et al., 2006; DI MICHELE et al., 2012; CAMPOS et al., 2014). Porém, seria necessário cada autor caracterizar a amostra com a especialidade de provas dos atletas estudados, assim como no estudo de Skorski et al. (2012), em que os autores dividiram os nadadores de acordo com distância da prova (velocidade e fundo), com a finalidade de melhor identificação do LAn.

Ao descrever a tabela de qualidade dos artigos, apenas seis obtiveram pontuação máxima, pois descrevem melhor seus estudos de acordo com os critérios que foram adotados (CAMPOS et al., 2014; SKORSKI et al., 2012; DI MICHELE et al., 2012; HOEFELMANN et al., 2011; DEMINICE et al., 2007; RIBEIRO et al., 2004). Nos demais artigos, determinados critérios deixaram de pontuar (KOKUBUN et al., 1996; PAPOTI et al., 2001; PYNE, LEE, SWANWICK, 2001; DENADAI et al., 2002; DEKERLE et al., 2002; GATTI et al., 2004; PAPOTI et al., 2005; LIMA et al., 2006; ANDERSON et al., 2006; MACHADO et al., 2011; KALVA-FILHO et al., 2015). Porém, eles apresentam bons métodos de identificação do LAn, podendo ser utilizados na área prática com atletas de natação. 


\section{Conclusão}

Conclui-se que é possível utilizar diferentes métodos para identificação do LAn e empregá-los na prescrição e controle do treinamento de nadadores. O protocolo de $200 \mathrm{~m}$ foi o mais utilizado, indicando ser o mais adequado para grupos de provas distintas. No entanto, a literatura ainda carece delineamentos adequados para cada estilo de nado, bem como para a especialidade de cada atleta.

\section{Referências}

ALEXIOU, S. et al. Lactate kinetics and performance in marathon swimming. Medical Science Research, v. 26, n. 3, p. 203-205, 1998.

ANDERSON, M. et al. Monitoring seasonal and long-term changes in test and competitive performance in elite swimmers. Journal of Science and Medicine in Sport. Elsevier, v. 6, n. 4, Supplement 1, p. 84-84, 2003.

ANDERSON, M. E. et al. Monitoring seasonal and long-term changes in test performance in elite swimmers. European Journal of Sport Science, v. 6, n. 3, p. 145-154, 2006.

AVLONITOU, E. Maximal lactate values following competitive performance varying according to age, sex and swimming style. The Journal of Sports Medicine and Physical Fitness, v. 36, n. 1, p. 24-30, 1996.

BARON, B.; PELAYO, P. Blood lactate, heart rate and systolic blood pressure measurements provide a reliable estimate of the aerobic capacity in running and swimming. Journal of Human Movement Studies, v. 41, n. 3, p. 165-174, 2001.

CAMPOS, E. Z. et al. Effects of Sodium Bicarbonate Ingestion during an Intermittent Exercise on Blood Lactate, Stroke Parameters, and Performance of Swimmers. Journal of Exercise Physiology Online, v. 15, n. 6, 2012.

CAMPOS, E. Z. et al. The response of the lactate minimum test to a 12 -week swimming training. Motriz: revista de Educação Física, Rio Claro, SP, v. 20, n. 3, p. 286-291, 2014.

CAPUTO, F. et al. Efeitos de oito semanas de treinamento de natação no limiar anaeróbio determinado na piscina e no ergômetro de braço. Revista Brasileira de Medicina do Esporte, São Paulo, v. 8, n. 1, p. 7-12, 2002.

CELLINI, M. et al. Noninvasive determination of the anaerobic threshold in swimming. International Journal of Sports Medicine, v. 7, n. 06, p. 347-351, 1986.

CHATARD, J.C.; MUJIKA, I. Training load and performance in swimming. Biomechanics and Medicine in Swimming VIII, v. 9, p. 429-434, 1999. 
DOI 10.5216/rpp.v22.49298

CLEMENTE-SUÁREZ, V.J. et al. The effects of two different swimming training periodization on physiological parameters at various exercise intensities. European Journal of Sport Science, v. 17, n. 4, p. 425-432, 2017.

COOK, D. J.; MULROW, C. D.; HAYNES, R. B. Systematic reviews: synthesis of best evidence for clinical decisions. Annals of Internal Medicine, v. 126, n. 5, p. 376-380, 1997.

COSTA, M. J. et al. Tracking the performance of world-ranked swimmers. Journal of Sports Science \& Medicine, v. 9, n. 3, p. 411, 2010.

COSTA, M. J. et al. Tracking the performance, energetics and biomechanics of international versus national level swimmers during a competitive season. European Journal of Applied Physiology, v. 112, n. 3, p. 811-820, 2012.

DA SILVA, S.F. et al. Comparação de método invasivo e não invasivo para determinação da velocidade crítica em nadadores. Revista de Educação Física/Journal Of Physical Education, v. 76, n. 136, 2007.

DEKERLE, J. et al. Validity and reliability of critical speed, critical stroke rate, and anaerobic capacity in relation to front crawl swimming performances. International Journal of Sports Medicine, v. 23, n. 2, p. 93-98, 2002.

DEMINICE, R. et al. Série de treinamento intervalado de alta intensidade como índice de determinação da tolerância à acidose na predição da performance anaeróbia de natação. Revista Brasileira de Medicina do Esporte, v. 13, n. 3, p. 185-189, 2007.

DI MICHELE, R. et al. Estimation of the anaerobic threshold from heart rate variability in an incremental swimming test. The Journal of Strength \& Conditioning Research, v. 26, n. 11, p. 3059-3066, 2012.

ESPADA, M. C. et al. Ventilatory and physiological responses in swimmers below and above their maximal lactate steady state. The Journal of Strength \& Conditioning Research, v. 29, n. 10, p. 2836-2843, 2015.

FAUDE, O.; KINDERMANN, W.; MEYER, T. Lactate threshold concepts. Sports Medicine, v. 39, n. 6, p. 469-490, 2009.

GACESA, J. Z. P.; BARAK, O. F.; GRUJIC, N. G. Maximal anaerobic power test in athletes of different sport disciplines. The Journal of Strength \& Conditioning Research, v. 23, n. 3, p. 751-755, 2009.

GATTI, R. G. O. et al. Respostas fisiológicas e biomecânicas de nadadores em diferentes intensidades de nado. Rev. Bras. Cineantropom. Desempenho Hum., Florianópolis, v. 6, n. 1, p. 26-35, 2004. 
DOI 10.5216/rpp.v22.49298

GOBATTO, C. A. et al. Maximal lactate steady state in rats submitted to swimming exercise. Comparative Biochemistry and Physiology Part A: Molecular \& Integrative Physiology, v. 130, n. 1, p. 21-27, 2001.

GOMES, A. L. M.; DANTAS, E. H. M.; CAMERON, L. C. Respostas fisiológicas e mecânicas do treinamento intervalado, de alta intensidade, de distâncias curtas a longas em atletas de natação. Fitness \& Performance Journal, Rio de Janeiro, v. 2, n. 2, p. 75-81, 2003.

GONDIM, F. J. et al. Determination of the anaerobic threshold and maximal lactate steady state speed in equines using the lactate minimum speed protocol. Comparative Biochemistry and Physiology Part A: Molecular \& Integrative Physiology, v. 146, n. 3, p. 375-380, 2007.

GRAEF, F. I.; KRUEL, L. F. M. Frequência cardíaca e percepção subjetiva do esforço no meio aquático: diferenças em relação ao meio terrestre e aplicações na prescrição do exercício - uma revisão. Revista Brasileira de Medicina do Esporte. São Paulo: SBME, v. 12, n. 4, p. 221-227, jul. 2006.

GRECO, C. C. et al. Efeitos do desempenho aeróbio na máxima fase estável de lactato sanguíneo determinada em protocolo intermitente na natação. Revista Brasileira de Medicina do Esporte, São Paulo, p. 130-133, 2010.

GREENWOOD, J. D. et al. Intensity of exercise recovery, blood lactate disappearance, and subsequent swimming performance. Journal of Sports Sciences, v. 26, n. 1, p. 29-34, 2008.

HOEFELMANN, L. P. et al. Comparação de diferentes métodos para identificação do limiar anaeróbio em nadadores. Journal of Physical Education, v. 22, n. 2, p. 297-305.

KALVA-FILHO, C. A. et al. Relationship of aerobic and anaerobic parameters with $400 \mathrm{~m}$ front crawl swimming performance. Biology of Sport, v. 32, n. 4, p. 333, 2015.

KOKUBUN, E. Velocidade crítica como estimador do limiar anaeróbio na natação. Rev. Paul. Educ. Fís, São Paulo, v. 10, n. 1, p. 5-20, 1996.

KONSTANTAKI, M.; SWAINE, I. L. Lactate and cardiopulmonary responses to simulated arm-pulling and leg-kicking in collegiate and recreational swimmers. International Journal of Sports Medicine, v. 20, n. 02, p. 118-121, 1999.

LI, Y. et al. Biochemical changes and endocrine responses in pre-competition training in elite swimmers. Biology of Sport, v. 29, n. 1, 2012.

LIMA, M. C. S. et al. Proposta de teste incremental baseado na percepção subjetiva de esforço para determinação de limiares metabólicos e parâmetros mecânicos do nado livre. Revista Brasileira de Medicina do Esporte, São Paulo, v. 12, n. 5, p. 268-274, 2006. 
DOI 10.5216/rpp.v22.49298 LONGO, C.; ALMEIDA, J. P.; ÃVILA, E. R. Limiar anaeróbico: comparação entre os métodos directos e indirectos em nadadores de alta competição Portugueses (+). Revista Portuguesa de Pneumologia, v. 1, n. 1, p. 53-58, 1995.

MACHADO, M. V. et al. Effect of 12 weeks of training on critical velocity and maximal lactate steady state in swimmers. European Journal of Sport Science, v. 11, n. 3, p. 165-170, 2011.

MAGLISCHO, E. W. Nadando ainda mais rápido: padrão de referência para o nadador profissional. São Paulo: Manole, 1999.

SAMPAIO, R. F. Estudos de revisão sistemática: um guia para síntese criteriosa da evidência científica. Revista Brasileira de Fisioterapia, São Paulo, v. 11, n. 1, p. 83-89, 2007.

MANCINI, M. C.; SAMPAIO, R. F. Estudos de revisão sistemática: um guia para síntese criteriosa da evidência científica. Revista Brasileira de Fisioterapia, São Paulo, v. 11, n. 1, p. 83-9, 2007.

MEJIAS, J. E. et al. "Young" masters vs. elite swimmers: comparison of performance, energetics, kinematics and efficiency. International Sport Med Journal. v. 15, n. 2, p. 165-177, 2014.

MERCIER, B. et al. Anaerobic and aerobic components during arm-crank exercise in sprint and middle-distance swimmers. European Journal of Applied Physiology and Occupational Physiology, v. 66, n. 5, p. 461-466, 1993.

NEIVA, H. P.; FERNANDES, R. J.; VILAS-BOAS, J. P. Anaerobic critical velocity in four swimming techniques. International Journal of Sports Medicine, v. 32, n. 03, p. 195-198, 2011.

OLBRECHT, J. Lactate production and metabolism in swimming. In: World Book of Swimming: from Science to Performance. New York: Nova, p. 255-276, 2011.

PAPOTI, M. et al. Aerobic and anaerobic performances in tethered swimming. International journal of sports medicine, v. 34, n. 08, p. 712-719, 2013.

PAPOTI, M. et al. Utilização do intercepto-yna avaliação da aptidão anaeróbia e predição da performance de nadadores treinados. Rev. Bras. Med. Esporte. v.11, n.2, p.126-130, 2005.

PAZA, D. L. S. et al. Cortisol capilar como medida de análise do estresse crônico. Psicologia, saúde \& doenças. v. 18, n.3, p.773-788, 2017.

PEREIRA, R. R. et al. Validação de dois protocolos para determinação do limiar anaeróbio em natação. Motriz, São Paulo, v. 8, p. 63-8, 2002. 
PYNE, D. B.; LEE, H.; SWANWICK, K. M. Monitoring the lactate threshold in worldranked swimmers. Medicine and Science in Sports and Exercise, v. 33, n. 2, p. 291-297, 2001.

REILLY, T.; WOODBRIDGE, V. Effects of moderate dietary manipulations on swim performance and on blood lactate-swimming velocity curves. International Journal of Sports Medicine, v. 20, n. 02, p. 93-97, 1999.

RIBEIRO, L. F. P. et al. Limiar anaeróbio em natação: comparação entre diferentes protocolos. Revista Brasileira de Educação Física e Esporte, São Paulo, v. 18, n. 2, p. 201-212, 2004.

RIBEIRO, L. F. P.; GALDINO, R.; BALIKIAN, P. Resposta lactacidêmica de nadadores e triatletas em função da utilização de "esteira" durante natação em velocidade correspondente ao limiar anaeróbio. Revista Paulista de Educação Física, São Paulo, v. 15, n. 1, p. 55-62, 2017.

SANTHIAGO, V. et al. Effects of 14-week swimming training program on the psychological, hormonal, and physiological parameters of elite women athletes. The Journal of Strength \& Conditioning Research, v. 25, n. 3, p. 825-832, 2011.

SANTHIAGO, V. et al. Responses of hematological parameters and aerobic performance of elite men and women swimmers during a 14-week training program. The Journal of Strength \& Conditioning Research, v. 23, n. 4, p. 1097-1105, 2009.

SANTOS, P. B.et al. A necessidade de parâmetros referenciais de cortisol em atletas: uma revisão sistemática. Motricidade, Curitiba, v. 10, n. 1, 2014.

SKORSKI, S. et al. Intensity control in swim training by means of the individual anaerobic threshold. The Journal of Strength \& Conditioning Research, v. 26, n. 12, p. 3304-3311, 2012.

SMITH, B. W.; MCMURRAY, R. G.; SYMANSKI, J. D. A comparison of the anaerobic threshold of sprint and endurance trained swimmers. The Journal of sports medicine and physical fitness, v. 24, n. 2, p. 94-99, 1984.

SOARES, S. M. et al. Assessment of Fatigue Thresholds in 50-m All-Out Swimming. International Journal of Sports Physiology and Performance, v. 9, n. 6, p. 959-965, 2014.

SOULTANAKIS, H. N.; MANDALOUFAS, M. F.; PLATANOU, T. I. Lactate threshold and performance adaptations to 4 weeks of training in untrained swimmers: volume vs. intensity. The Journal of Strength \& Conditioning Research, v. 26, n. 1, p. 131-137, 2012. 
DOI 10.5216/rpp.v22.49298

TOUBEKIS, A. G.; TOKMAKIDIS, S. P. Metabolic responses at various intensities relative to critical swimming velocity. The Journal of Strength \& Conditioning Research, v. 27, n. 6, p. 1731-1741, 2013.

TREFFENE, R. J. et al. Lactic acid accumulation during constant speed swimming at controlled relative intensities. The Journal of sports medicine and physical fitness, v. 20, n. 3, p. 244-254, 1980.

WELTMAN, A. The blood lactate response to exercise. Champaign: Human Kinetics, 1995.

ZOLTOWSKI, A. P. C. et al. Qualidade metodológica das revisões sistemáticas em periódicos de psicologia brasileiros. Psicologia: teoria e pesquisa, Rio Grande do Sul, v. 30, n. 1, p. 97-104, 2014.

Recebido em: 11/09/2017

Revisado em: 08/02/2018

Aprovado em: 04/09/2019

Endereço para correspondência:

alysson.enes@hotmail.com

Alysson Afonso Nadalin Enes

Universidade Federal do Paraná, Departamento de Educação Física.

Rua Coração de Maria, 92

Jardim Botânico

80210132 - Curitiba, PR - Brasil 